\title{
Suitability System of Microbiological Method for Nystatin Potency Determination in the Routine Analysis Using Agar Diffusion Method
}

\author{
Dalia Essam Eissa ${ }^{1}$, Engy Refaat Rashed ${ }^{2}$, Mostafa Essam Eissa ${ }^{3 *}$ \\ ${ }^{I}$ Royal Oldham Hospital, Oldham, United Kingdom. \\ ${ }^{2}$ National Centre for Radiation Research and Technology, Cairo, Egypt. \\ ${ }^{3}$ Independent Researcher, Faculty of Pharmacy, Cairo University, Cairo, Egypt.
}

Received 25 March 2021; Revised 21 June 2020; Accepted 11 October 2021; Published 01 December 2021

\begin{abstract}
Nystatin is a polyene macrolide antifungal active which is used for the treatment of candidiasis and obtained from some species of Streptomyces bacteria. The present work describes the statistical suitability analysis for regular monitoring of the agar diffusion bioassay in a simple, inexpensive and time-saving process before potency determination. A balanced (symmetrical) two-dose parallel line assay model was applied using the agar well diffusion method for quantification of Nystatin in raw material and finished medicinal dosage form. The routine inspection methodology yielded good results and included calculations by the linear parallel model and by means of regression analysis and verified using analysis of variance (ANOVA). The assay is based on the inhibitory effect of Nystatin upon a standard strain as described in the United States Pharmacopeia (USP). The results of the post validation regular assays were treated statistically by ANOVA and the deviations (expressed as average \pm standard deviation) from both raw and column totals were $0.702 \pm 0.476$ and $0.865 \pm 0.468$, respectively. The mean value of the variance ratio for regression and parallelism squares were $534.349 \pm$ 212.546 and $0.596 \pm 0.345$, respectively. The study of Nystatin's ongoing analysis showed that the microbiological assay design is satisfactory with respect to the limiting values for the determination of the potency. The established balanced parallel line assay is reasonably stable and suitable and can be used for the regular drug analysis in routine quality control testing and the quantitation of Nystatin in pharmaceutical dosage form and raw material.
\end{abstract}

Keywords: Nystatin; parallel line assay; quality control; agar diffusion; regression; parallelism.

\section{Introduction}

Nystatin is referred to as the polyene macrolide antifungal compound, which could be obtained from some species of Streptomyces (Streptomyces noursei) bacteria and is used for the treatment of different types of candidiasis [1, 2]. It is prescribed to treat Candida infections of the human skin such as diaper rash, thrush, oropharyngeal and mucous membrane (non-oesophageal) GI candidiasis, and vaginal yeast infections [3-5]. It may also be used to prevent candidiasis in high-risk populations [6, 7]. Nystatin may be used by mouth, in the vagina, or applied to the skin [1]. Nystatin IUPAC designation is detailed as (1S,3R,4R,7R,9R,11R,15S,16R,17R, 18S, 19E, 21E, 25E, 27E, 29E,31E, 33R,35S,36R,37S)-33-[(2R,3S,4S,5S,6R)-4-amino-3,5-dihydroxy-6-methyloxan-2-yl]oxy-1,3,4,7,9,11,17,37-octahydr oxy-15,16,18-trimethyl-13-oxo-14,39-dioxabicyclo [33.3.1] nonatriaconta-19,21,25,27,29,31-hexaene-36-carboxylic acid (Figure 1) [8]. The chemical structure features a large ring of atoms (in essence, a cyclic ester ring) containing

* Corresponding author: mostafaessameissa@yahoo.com

$>$ This is an open access article under the CC-BY license (https://creativecommons.org/licenses/by/4.0/).

(C) Authors retain all copyrights. 
multiple conjugated carbon-carbon $(-\mathrm{C}=\mathrm{C}-$ ) double bonds (accordingly, polyene) on one side of the ring and multiple hydroxyl groups bonded to the other side of the ring. The structure also often has a d-mycosamine (a type of aminoglycoside) group bonded to the molecule [9]. The series of conjugated double bonds typically absorbs strongly in the ultraviolet-visible region of the electromagnetic spectrum, often resulting in the polyene antibiotics having a yellow colour $[10,11]$. The activity of this antifungal drug is determined using a microbiological assay technique.

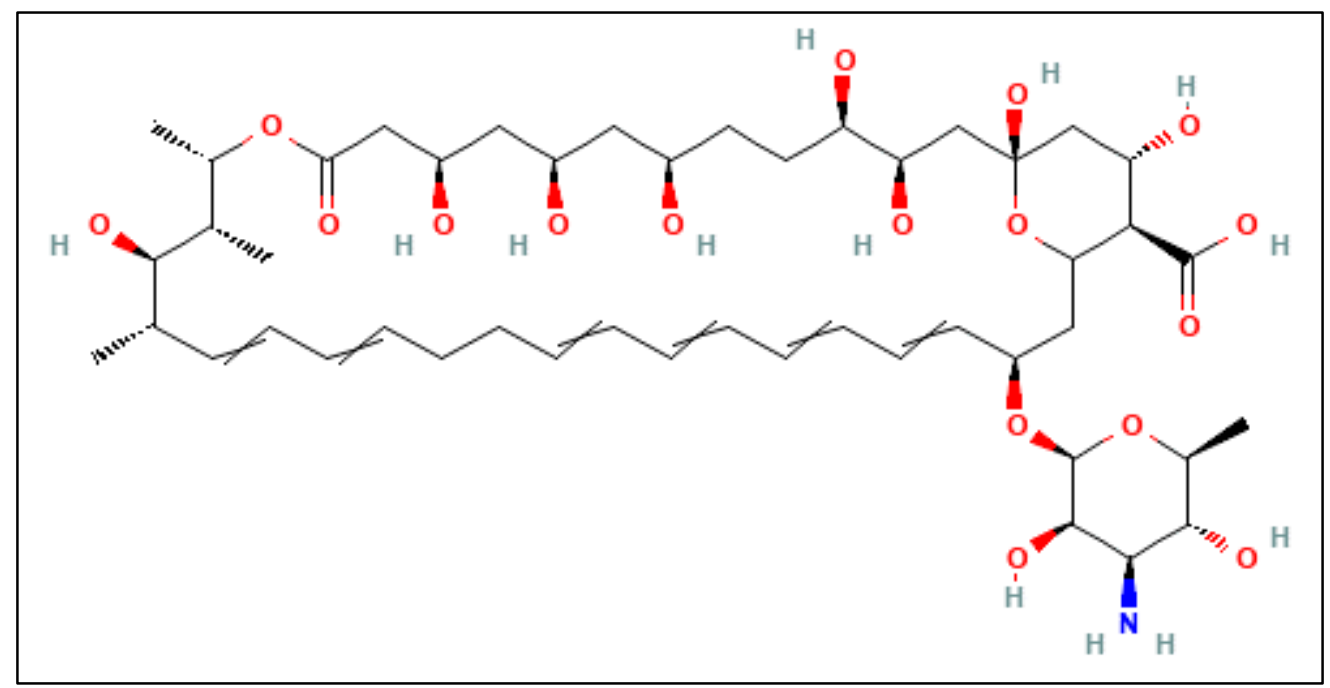

Figure 1. The structure of polyene antifungal (antimycotic) compound Nystatin

The strength of antimicrobial materials can be determined by both chemical and biological techniques [12]. These methods embrace microbiological assays, automated chemical assays (such as high or ultrahigh-performance liquid chromatography (HPLC or UPLC)), immunological assays (e.g. fluorescence polarization immune assay, fluorescence immunoassay) and radioimmunoassay [13]. However, the analysis methodology has been transformed to chromatographic assays for many antibiotics as they provide quantitative measurements of the purity and the amounts and types of impurities in antibiotics. Nevertheless, a significant number of commercially and medicinally important antibiotics are subject to analysis by microbiological assays till now [14]. The determination of antibiotic components is usually done using chemical means such as HPLC or UPLC and ultraviolet (UV) spectrophotometry, but these methods cannot provide a true indication of biological activity which is the main disadvantage of these methods. However, the microbiological assay can precisely determine both the potency and bioactivity of an antibiotic. Besides, the microbiological assay does not require specialized equipment or toxic diluents, buffers and mobile phases [15, 16]. Impurities and related substances do not affect the results of the microbiological assay [17]. It also precisely quantifies the actual concentration of the active ingredient in antibiotic preparation when a microbial resistant situation arises. The microbiological method is the most convenient way to determine the potency of antibiotics [18].

Since the microbiological assay is still holding its critical position for the evaluation of the antimicrobial materials (from the previous argument paragraph), the validity and the quality of the results derived from the test must be ensured to assure the conformance of the product to its dedicated use. Determination of antimicrobial potency is extremely important for the quality control and quality assurance of antibiotic preparations. Hence, it is necessary to select a practical and economical method for the quality control of antibiotics $[19,20]$. Recently an implementation of microbiological analysis has been developed for parenteral antibiotics by intravenous route of administration. This biological assay is widely acceptable by regulatory authorities to monitor antibiotic potency [21]. Being a biological assay, the microbiological assay is subject to some biological errors, but after validating all variable parameters it is possible to obtain meaningful results and to achieve a precision similar to that of many chemical methods [17, 22]. Microbiological assay in comparison to chemical methods measures the true response of antibiotics on the biological system and is used to obtain a more realistic and precise measurement of potency to overcome the antibiotic resistance problem [12, 22-24].

In presence of challenging situations of a growing number of populations with weak and compromised immune systems, there is an utmost necessity for antimicrobial agents to combat various types of infections. The delivered antibiotic medications to the patients must be ensured to be of high quality and safety. Thus, the prescribed doses must be met with high accuracy and confidence based on the labelled concentration of the active pharmaceutical ingredients (APIs). The present study herein presents a fast, simple, inexpensive system for assessing the validity of routine microbiological assay in the post-validation phase using an Excel-based template that has been prepared in advance and validated before implementation which will be helpful for analysts in microbiology laboratories, especially in developing countries and poor nations who would need to determine the validity of their analysis for the potencies estimations before calculation of the strengths or activities of the antimicrobial entity. 


\section{Material and Methods}

\subsection{Microbiological Test Evaluation System}

Linear parallel model and regression analysis were used for statistical assay calculations [18]. Verification was performed using analysis of variance (ANOVA) [18]. The statistical and potency calculations described by Hewitt and European Pharmacopoeia Commission (EPC) were applied to the results of a microbiological assay of the antibiotic Nystatin - that have comparable analysis design - as raw material and in medicinal preparation using the cylinder-plate method (parallel lines with two reference standards and unknowns' doses) [25, 26]. Initial investigation of datasets involves outliers' detection as individual recorded values and homogeneity of variances using Cochran test using manual formula entry in Excel or built-in program integrated with it. Figure 2 describes the layout of the process methodology as a flowchart used for the assay potency determinations. To evaluate the results, preliminary calculations are presented for the purpose of preparing datasets required to complete the results. Calculations and graphs were performed using Excel.

\subsection{Microbiological Analysis of Nystatin}

The basic analysis was performed as described in United States Pharmacopoeia and National Formulary (USP43NF38) [27]. The dilutions of the unknown (U), Nystatin test material and reference standards (S) were prepared at two concentration levels of (1) $10 \mu \mathrm{g} \mathrm{mL}-1$ and (2) $20 \mu \mathrm{g} \mathrm{mL}-1$. The well-known design of the $2 \times 2$ assay (known as symmetric or balanced analysis) was used in the present study [26]. Doses of standard and tests were placed once in each block (large rectangular $8 \times 8$ plate) by punching wells in the solidified two-layer antibiotic agar medium, up to a total of three test subjects were incorporated in a single plate. All reagents used were analytical grade. Purified water was used in all experiments [28]. Commercial products and raw Active Pharmaceutical Product (API) were obtained from the market retail.

\subsection{Trending of Assay Validity Parameters and Potencies}

Assay evaluation parameters were interpreted using control or trending charts performed by excel drawing to provide laboratory self-assessment for the consistency of the analysis operation. This is done along with the trend of estimated potencies for both raw material (RM) and finished product (FN) as International Unit (I.U.) per mg of powder and $\mathrm{g}$ of the medicinal product, respectively. The basic assay variabilities included were deviations from column total, deviations from the raw total, regression squares, parallelism and variations from preparation total. The normality of data trends could be checked either manually or through calculation in an Excel sheet. The applied type of the process-behavior chart was Individual-Moving Range (I-MR or XmR) plot. The principle of constructing and interpreting the milestone trending charts has been addressed previously in other works [29-32]. Equations that are necessary to construct the components of the control charts have been formulated in Excel or charts have been drawn directly through built-in programs.

\section{Results and Discussion}

The activity of antimicrobial agents may be demonstrated under suitable conditions by their inhibitory effect on microorganisms $[17,19]$. Although the microbiological assay methods involve many steps and are time-consuming, they can reveal subtle changes not demonstrable by chemical methods [19]. Data processing could be rendered easier and time-saving with minimal error using appropriate formulae that have been programmed in a worksheet [25, 26].

The Parallel Line Model (PLM) is one of the most important designs for biological assays. The currently used analysis belongs to Latin square assay of symmetrical type [33]. Since Nystatin is assigned to the WHO group B index of degradable substances, the microbiological assay is a crucial test for the determination of the true activity of the material as a pure entity and in pharmaceutical formulations. Importantly, the validity of the potencies obtained from the assay determinations must be verified through checking tests sources of variability quantitatively of which two of them are pharmacopeial, namely parallelism and regression analysis [25]. Accordingly, a spreadsheet has been adopted for the presumed assay design to be used for regular assay of Nystatin from which there are no outlier variances could be detected and the treatment groups passed the Cochran test [34]. The variance ratios (F-test) for the sources of the test variabilities were tested against a limiting value to evaluate the system suitability for the assay. Excel sheet template was evaluated against Hewitt and EPC prior to the implementation in the practical evaluation of ten tests performed for Nystatin raw materials and healthcare products [25, 26]. This was done in a previous study as a good practice strategy for programs and software validation before application in routine work activities. The recorded zone of inhibitions (in $\mathrm{mm} \times 10$ ) were tabulated in columns and the dispersion of each dataset was evaluated visually using box and whisker plot in Figure 3. The spreading pattern of data was acceptable approaching normal scatter. 
Table 1 demonstrates outlier detection and only highlighted cells were spotted as aberrant values. However, the automatic rejection rule was not recommended because after refereeing again to the previous figure, it was found that these treatments demonstrated a tight clustering pattern. Thus, an apparently normal value - known by the experience of the zone diameter trend - might appear as an excursion [25]. Accordingly, a decision was made to not exclude these values. The calculated parameters of experimental variability sources for the successive experiments were determined and recoded to build the cornerstone process-behavior charts. At $p=0.05$, the calculated limiting values derivatives from (row, column and preparation) totals, (regression and parallelism) and residual error (SSreg) squares were 2.24, $2.24,2.83,12.52,2.83$ and 1.67, respectively. The degree of freedom (d.f.) for the previous parameters, in addition to total (SStot) was seven, seven, seven, three, one, three, 42 and 63, respectively. This analysis is mandatory to judge the quality of the assay before the determination of the potency of the unknowns or test subjects.

\section{Preparation for antibiotic assay}

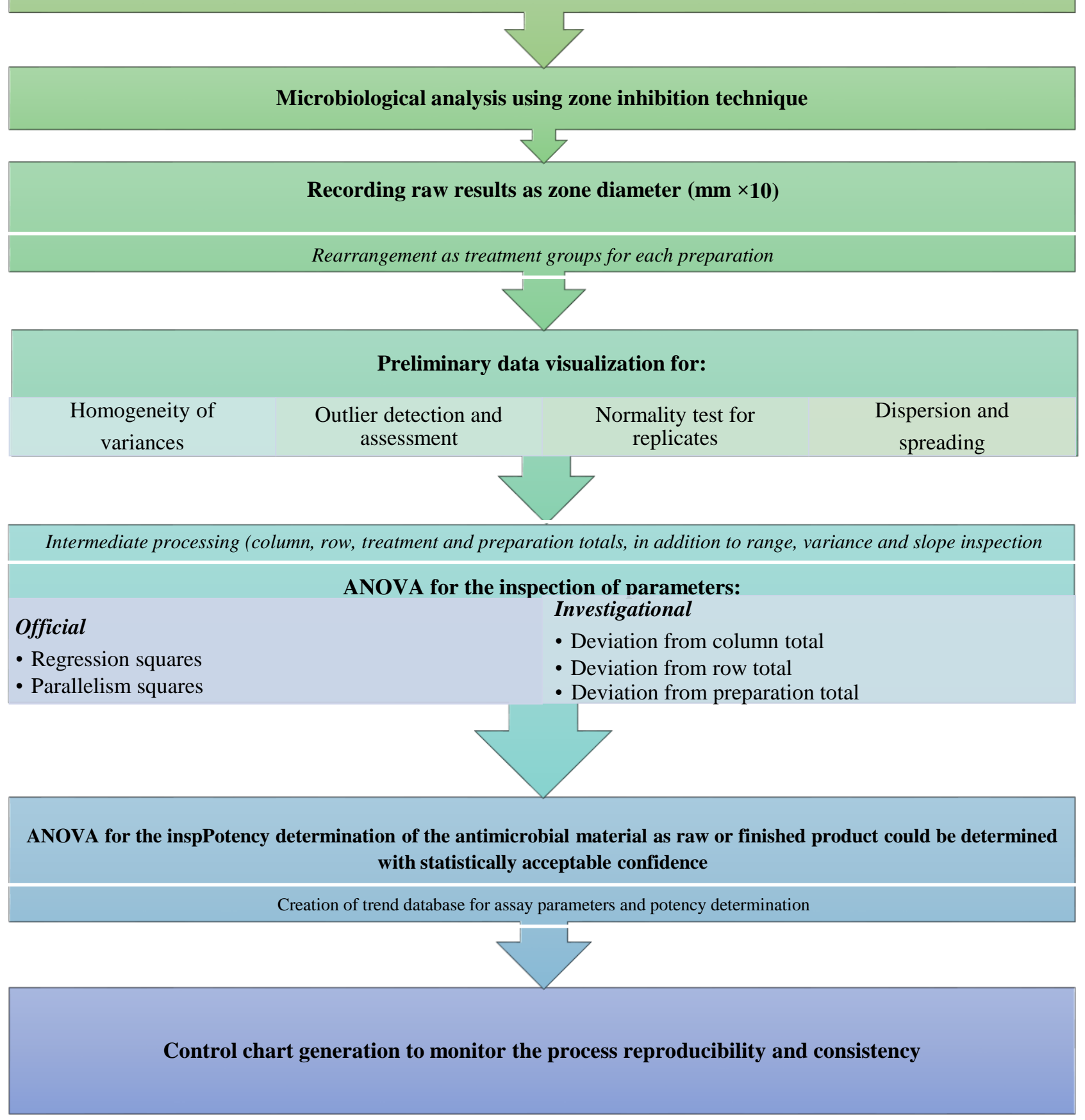

Figure 2. Flowchart showing research methodology for microbiological assay suitability and internal quality assessment for the analysis 

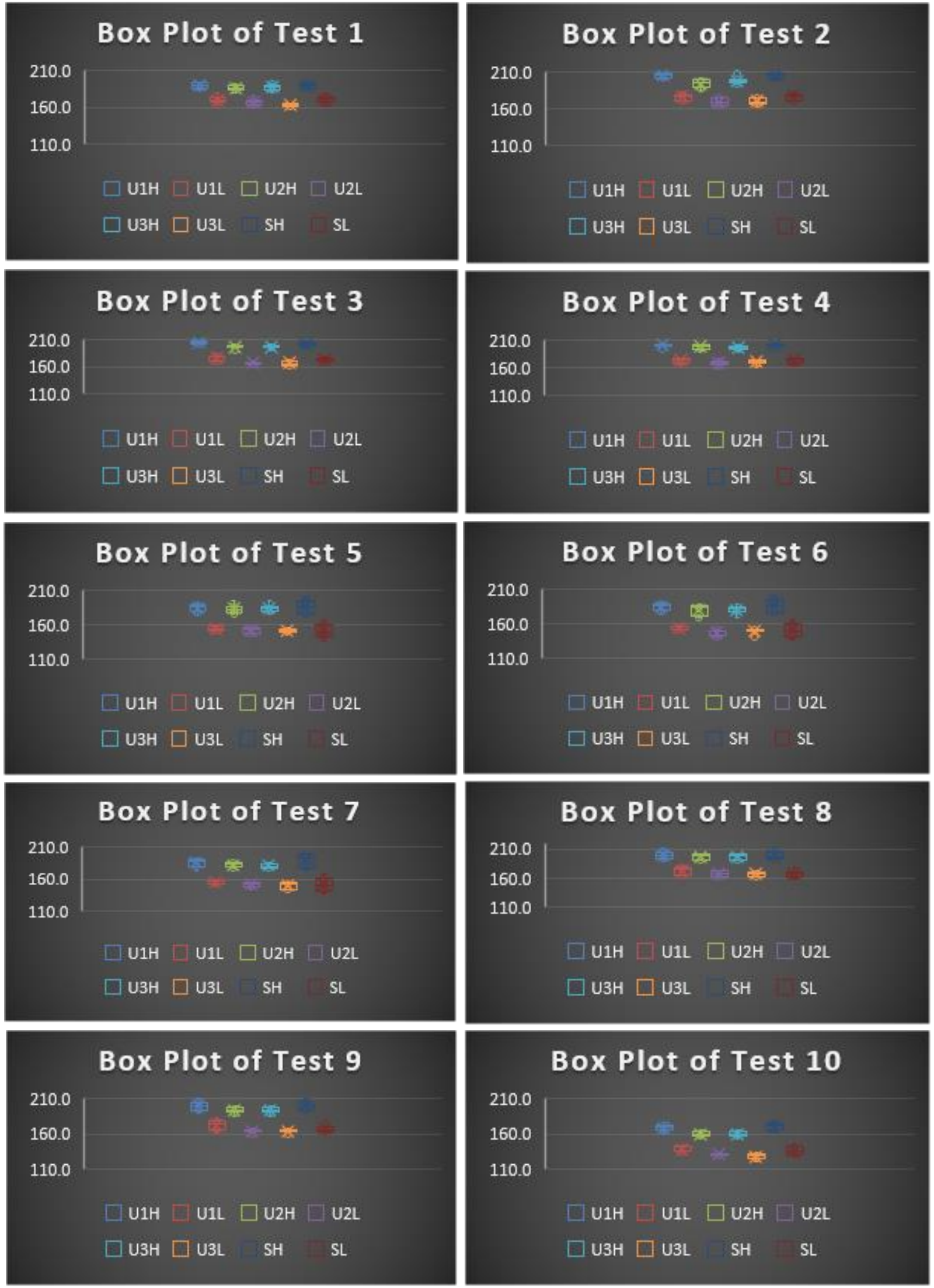

Figure 3. Box-and-Whisker diagram showing data dispersion pattern of the inhibition zone diameters ( $\mathrm{mm} x \mathrm{10})$ in each treatment groups of the microbiological assay plates of Nystatin 
Table 1. Outlier detection in dataset using USP method

\begin{tabular}{|c|c|c|c|c|c|c|c|c|c|}
\hline \multirow{2}{*}{\multicolumn{2}{|c|}{ Assay outlier test }} & \multicolumn{8}{|c|}{ Treatment groups/Plate (Limiting Value of $\left.\mathbf{G}_{2}=0.78\right)^{a}$} \\
\hline & & \multirow{2}{*}{$\begin{array}{c}\mathbf{U}_{\mathbf{1 H}} \\
0.241\end{array}$} & \multirow{2}{*}{$\frac{\mathbf{U}_{1 \mathrm{~L}}}{0.540}$} & \multirow{2}{*}{$\begin{array}{c}\mathbf{U}_{2 \mathrm{H}} \\
0.547\end{array}$} & \multirow{2}{*}{$\frac{\mathbf{U}_{2 \mathrm{~L}}}{0.519}$} & \multirow{2}{*}{$\begin{array}{c}\mathbf{U}_{\mathbf{3 H}} \\
0.406\end{array}$} & \multirow{2}{*}{$\frac{\mathbf{U}_{\mathbf{3 L}}}{0.025}$} & \multirow{2}{*}{$\begin{array}{c}\mathbf{S}_{\mathbf{H}} \\
0.350\end{array}$} & \multirow{2}{*}{$\begin{array}{l}\mathbf{S}_{\mathbf{L}} \\
0.636\end{array}$} \\
\hline USP R of & Upper Extreme & & & & & & & & \\
\hline Test 1 & Lower Extreme & 0.466 & 0.664 & 0.439 & 0.500 & 0.404 & $0.875^{b}$ & 0.552 & 0.374 \\
\hline \multirow{2}{*}{$\begin{array}{l}\text { USP R of } \\
\text { Test } 2\end{array}$} & Upper Extreme & 0.210 & 0.343 & 0.274 & 0.009 & 0.659 & 0.202 & 0.210 & 0.343 \\
\hline & Lower Extreme & 0.468 & 0.154 & 0.277 & 0.103 & 0.524 & 0.403 & 0.468 & 0.154 \\
\hline \multirow{2}{*}{$\begin{array}{l}\text { USP R of } \\
\text { Test } 3\end{array}$} & Upper Extreme & 0.250 & 0.343 & 0.657 & $0.838^{b}$ & 0.722 & 0.091 & 0.560 & 0.229 \\
\hline & Lower Extreme & 0.185 & 0.154 & 0.639 & 0.444 & 0.478 & 0.315 & 0.500 & 0.515 \\
\hline \multirow{2}{*}{$\begin{array}{c}\text { USP R of } \\
\text { Test } 4\end{array}$} & Upper Extreme & 0.457 & 0.229 & 0.152 & 0.393 & 0.469 & 0.200 & 0.457 & 0.229 \\
\hline & Lower Extreme & 0.713 & 0.515 & 0.365 & 0.319 & 0.245 & 0.731 & 0.713 & 0.515 \\
\hline \multirow{2}{*}{$\begin{array}{l}\text { USP R of } \\
\text { Test } 5\end{array}$} & Upper Extreme & 0.237 & 0.597 & 0.472 & 0.211 & 0.558 & 0.482 & 0.398 & 0.333 \\
\hline & Lower Extreme & 0.734 & 0.203 & 0.268 & 0.126 & 0.268 & 0.533 & 0.118 & 0.289 \\
\hline \multirow{2}{*}{$\begin{array}{l}\text { USP R of } \\
\text { Test } 6\end{array}$} & Upper Extreme & 0.237 & 0.597 & 0.141 & 0.310 & 0.238 & 0.375 & 0.398 & 0.333 \\
\hline & Lower Extreme & 0.734 & 0.203 & 0.407 & 0.745 & 0.474 & 0.690 & 0.118 & 0.289 \\
\hline \multirow{2}{*}{ USP R of Test 7} & Upper Extreme & 0.237 & 0.597 & 0.111 & 0.123 & 0.632 & 0.064 & 0.053 & 0.333 \\
\hline & Lower Extreme & 0.734 & 0.203 & 0.368 & 0.480 & 0.059 & 0.513 & 0.328 & 0.289 \\
\hline \multirow{2}{*}{$\begin{array}{l}\text { USP R of } \\
\text { Test } 8\end{array}$} & Upper Extreme & 0.524 & 0.220 & 0.290 & 0.020 & 0.290 & 0.250 & 0.587 & 0.664 \\
\hline & Lower Extreme & 0.463 & 0.361 & 0.209 & 0.113 & 0.209 & 0.121 & 0.178 & 0.312 \\
\hline \multirow{2}{*}{$\begin{array}{l}\text { USP R of } \\
\text { Test } 9\end{array}$} & Upper Extreme & 0.524 & 0.220 & 0.343 & 0.405 & 0.343 & 0.541 & 0.587 & 0.664 \\
\hline & Lower Extreme & 0.463 & 0.361 & 0.658 & 0.627 & 0.658 & 0.627 & 0.178 & 0.312 \\
\hline \multirow{2}{*}{$\begin{array}{l}\text { USP R of } \\
\text { Test } 10\end{array}$} & Upper Extreme & 0.455 & 0.398 & 0.235 & $0.933^{b}$ & 0.235 & 0.483 & 0.207 & 0.361 \\
\hline & Lower Extreme & 0.563 & 0.293 & 0.116 & 0.071 & 0.116 & 0.377 & 0.719 & 0.324 \\
\hline
\end{tabular}

${ }^{a}$ The implemented equation for detecting aberrant values; $\mathrm{G} 2=(\mathrm{y} 3-\mathrm{y} 1) /(\mathrm{yN}-1-\mathrm{y} 1)$, for $\mathrm{N}=8$ to 13 value/treatment or dose.

${ }^{\mathrm{b}}$ Red highlighted cells are excursions in one side of the extreme values.

Preliminary evaluation of the raw data obtained from the inhibition zone diameter of the large agar plates is an important part of good laboratory practice (GLP) to ensure the validity of the assay determinations. Basic assumptions concerning microbiological assay involve linearity of the response with the logarithm of dose over the range of the analysis doses using a randomized allocation of the treatments across the plate to compensate for any possible deviation or error and the responses are expected to be normally distributed. Moreover, the standard deviations of the treatment groups should not differ statistically from each other [25, 26]. This hypothesis was investigated, and the outcome confirmed in Figure 4 using the Cochran C test for detection of outlier variance(s) [35]. Nevertheless, normality analysis of the datasets was not of great concern in the microbiological assay as it is almost fulfilled. Furthermore, replication in any treatment would minimize deviations introduced in the analysis so that there will be no serious flaws in the test performed according to European Pharmacopoeia (EP) 2000 [36]. The present tests complied reasonably with the above requirements.

Table 2 shows the degree of conformance of the assessed trends with Gaussian distribution. Data analysis and interpretation were conducted using the same methodology described by the GraphPad manual which showed an acceptable level of normality at $\alpha=0.05$ except raw total variance ratio factor [37, 38]. However, it showed a reasonable level of normality at $\alpha=0.01$. This small deviation in normality is not of great concern because the implemented process-behavior chart - in Figures 5 to 7 - shows significant robustness against deviation from Gaussian dispersion [39, 40]. I-MR plot draws two charts: one for visualizing the variation in the inspection characteristics and the other for tracking the process mean [41]. All points are confined within the windows of the control limits where the Upper Control Limit (UCL) exceeded the critical limiting values for row and column totals, in addition to the preparation total deviations [42]. Appropriately designed Latin square to ensure the randomness of treatment groups for different preparations will minimize the impact of drifts from row and/or column totals [25, 26].

Table 2. Normality test for variances ratios and the potencies of Nystatin as a raw material and API in a medicinal product

\begin{tabular}{|c|c|c|c|c|c|c|c|}
\hline Anderson-Darling test & $\mathbf{R M}$ & $\mathbf{F N}$ & Parallelism Squares & $\begin{array}{l}\text { Raw } \\
\text { Total }\end{array}$ & $\begin{array}{c}\text { Preparation } \\
\text { Total }\end{array}$ & $\begin{array}{c}\text { Column } \\
\text { Total } \\
\end{array}$ & $\begin{array}{c}\text { Regression } \\
\text { Squares }\end{array}$ \\
\hline $\mathrm{A} 2 *$ & 0.4363 & 0.3486 & 0.3529 & 0.6872 & 0.5642 & 0.2583 & 0.4160 \\
\hline$P$ value & 0.2351 & 0.4226 & 0.3880 & 0.0497 & 0.1070 & 0.6332 & 0.2662 \\
\hline Passed normality test (alpha $=0.01) ?$ & Yes & Yes & Yes & Yes & Yes & Yes & Yes \\
\hline$P$ value summary & ns & ns & ns & $*$ & ns & ns & ns \\
\hline
\end{tabular}

ns: Not significant $\quad$ RM: Raw material $\quad$ FN; Finished product 

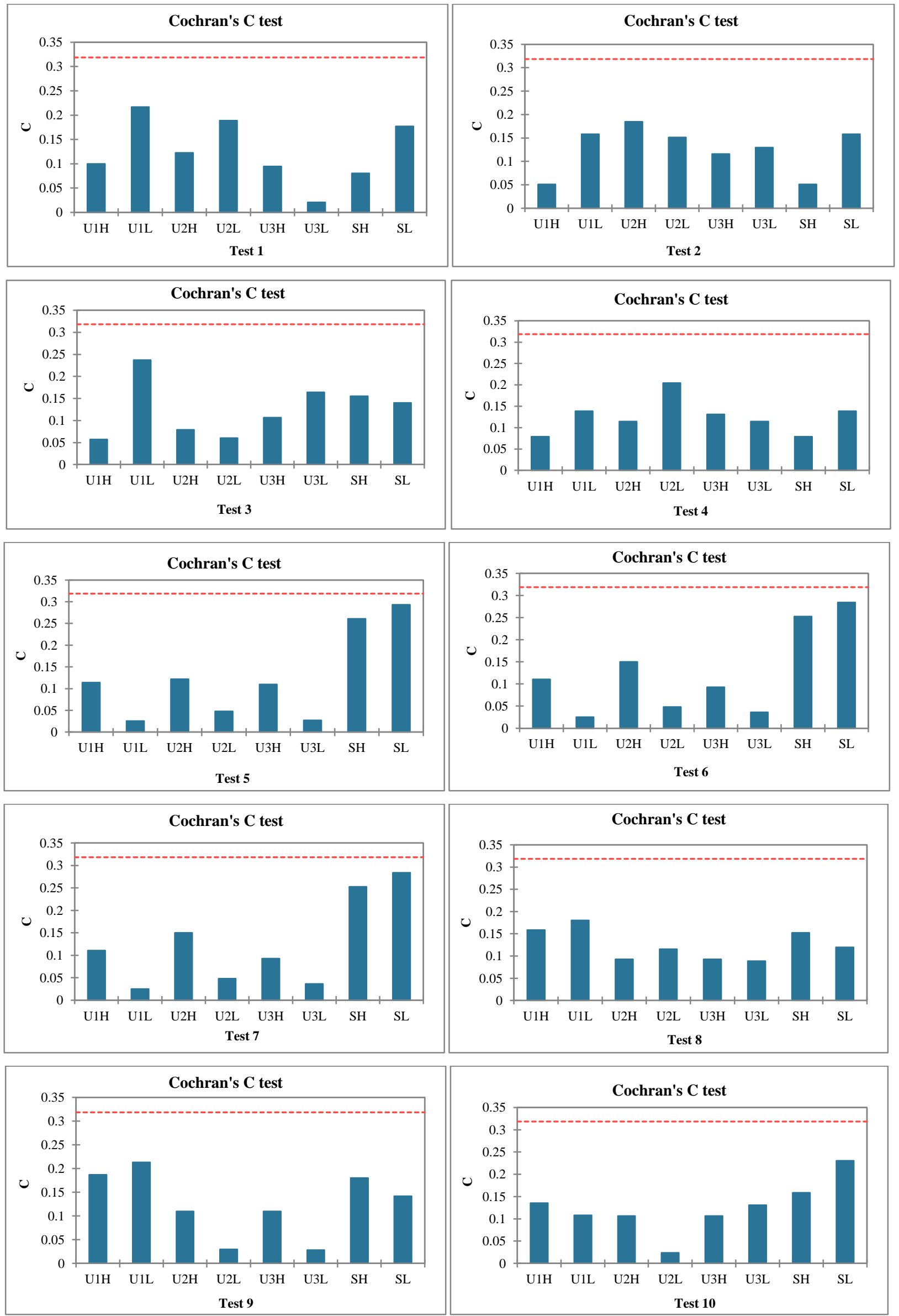

Figure 4. Cochran test for variance outlier detection in the treatment groups for ten microbiological assays of Nystatin 

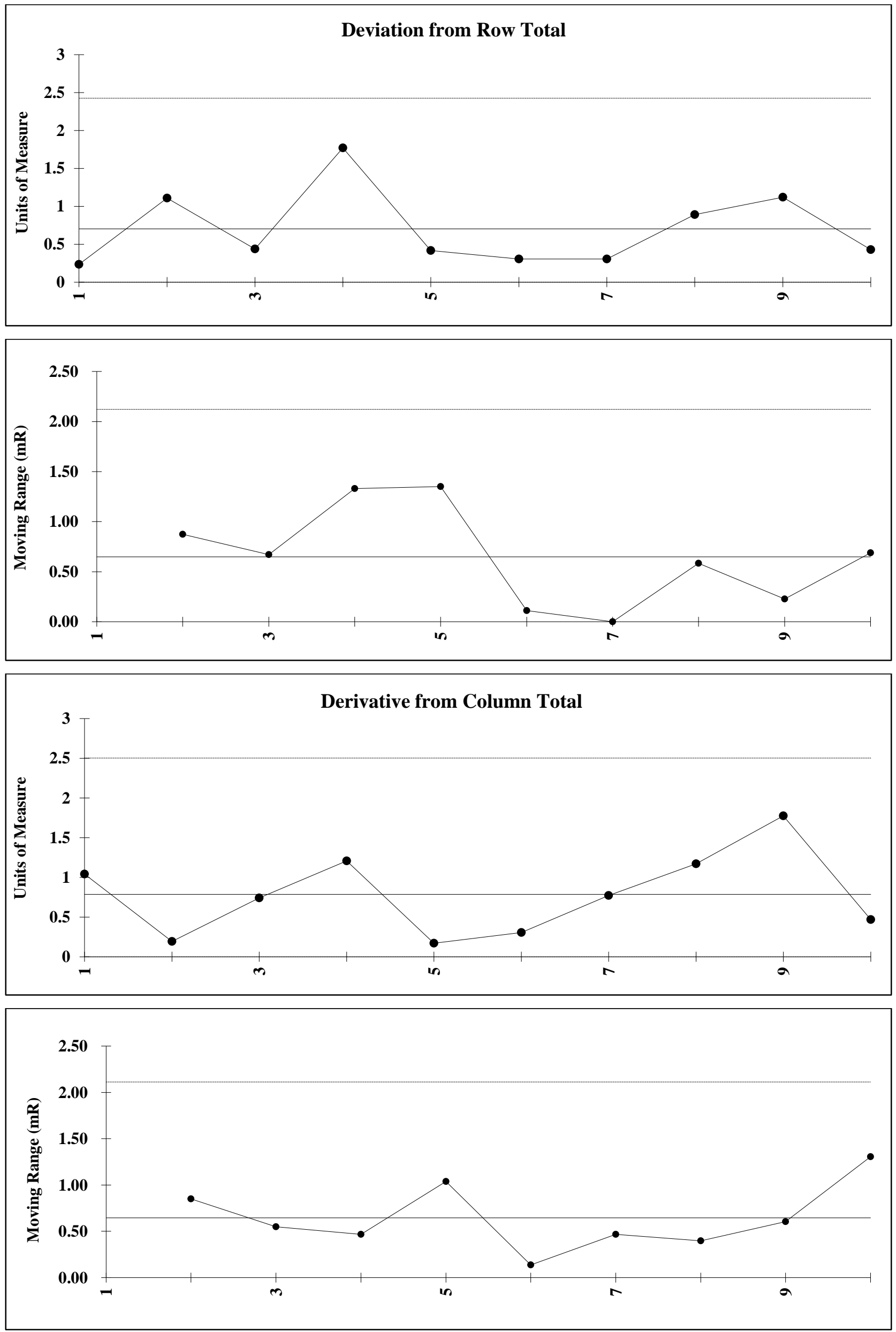

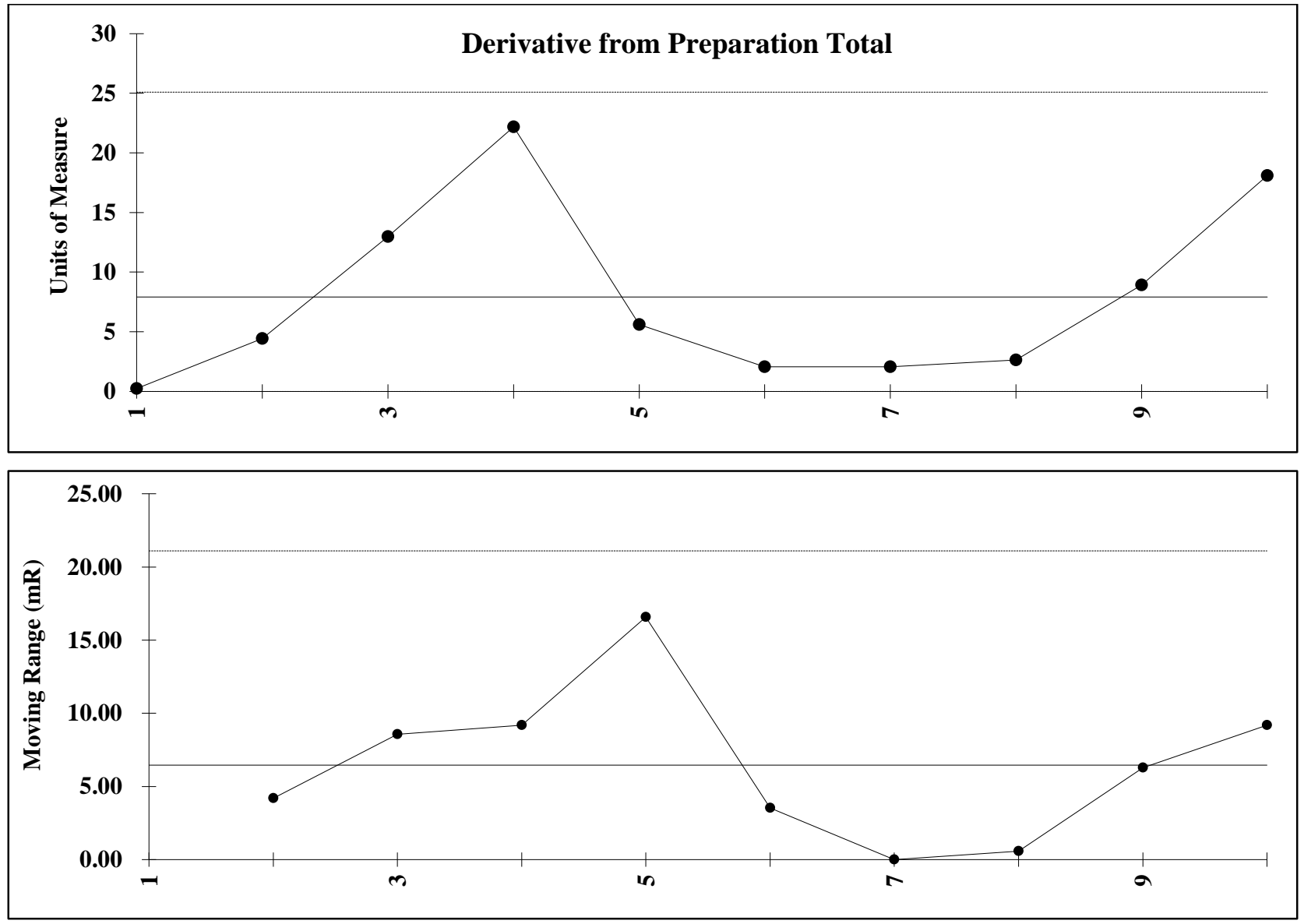

Figure 5. I-MR (XmR) control charts for laboratory self-assessment of the running microbiological assay suitability: row total, column total and preparation total
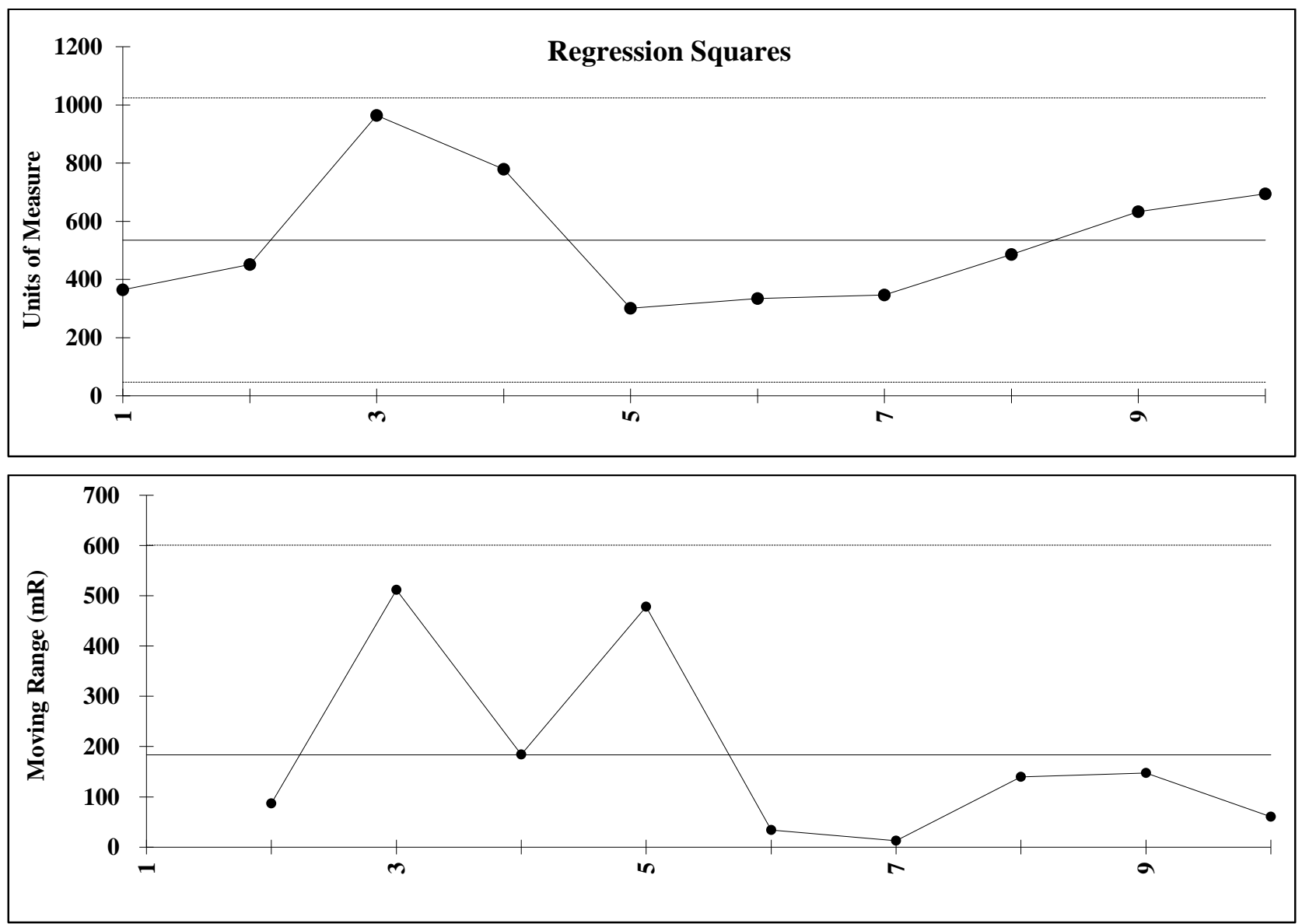

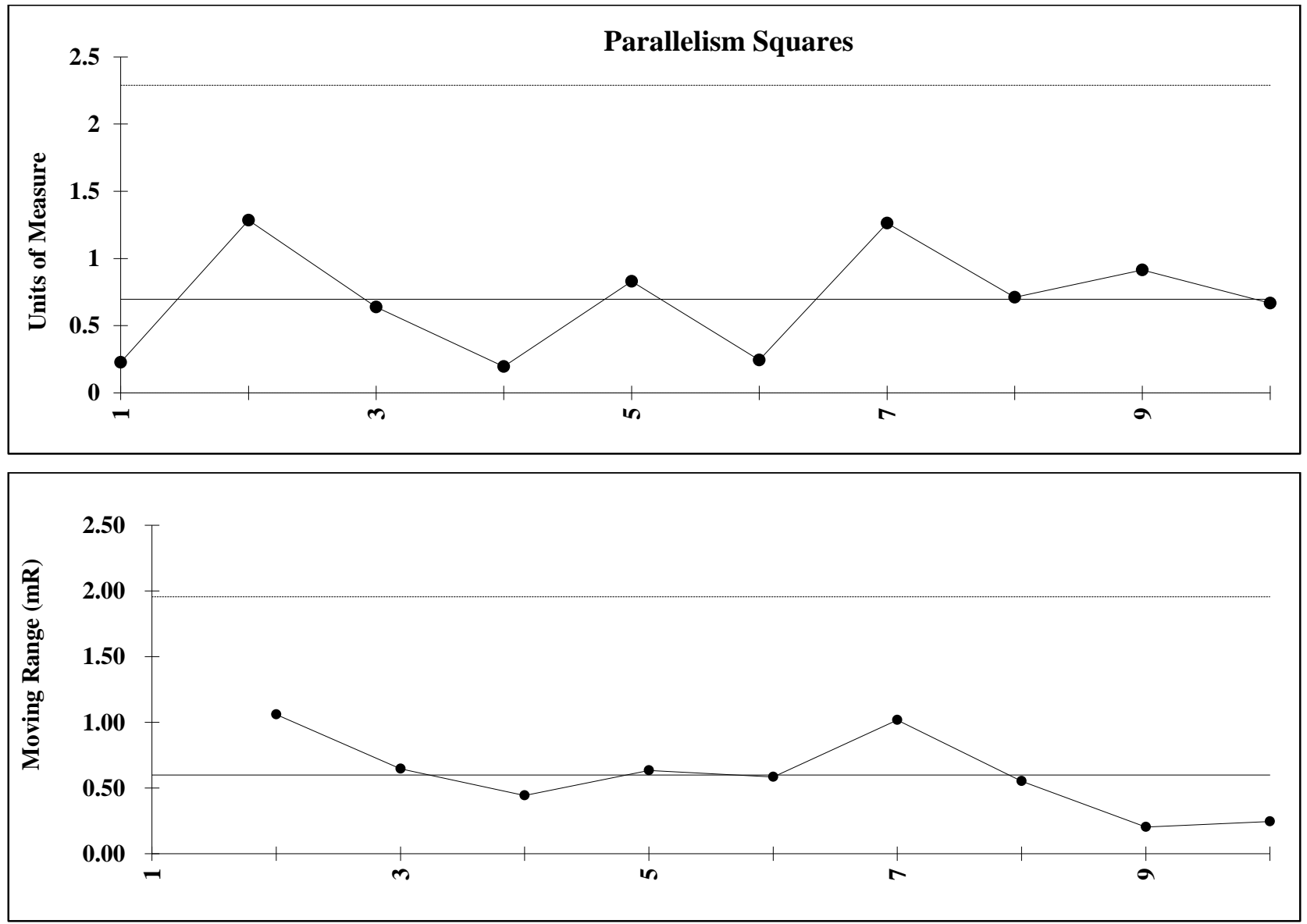

Figure 6. I-MR (XmR) control charts for laboratory self-assessment of the running microbiological assay suitability: regression squares and parallelism squares
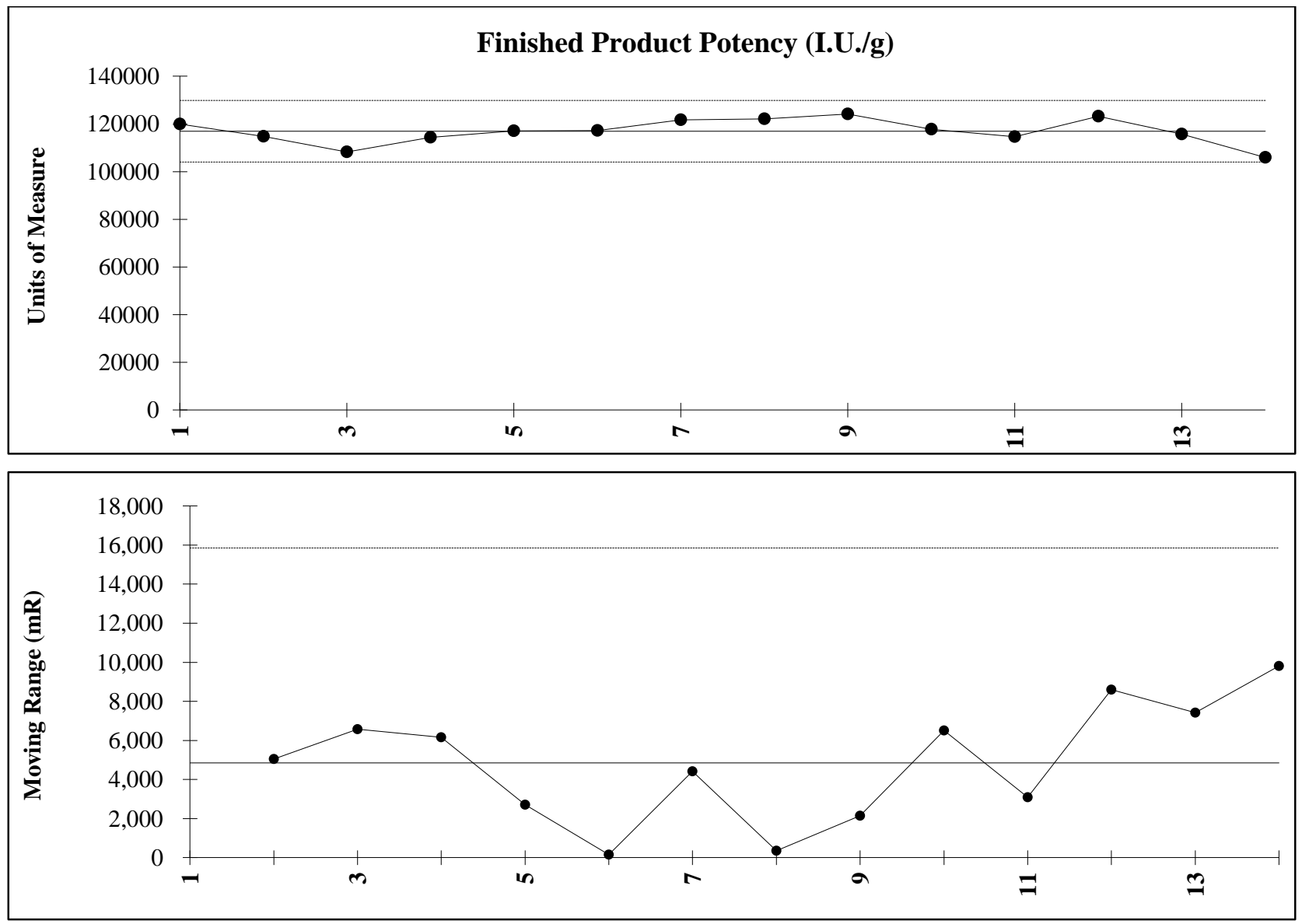

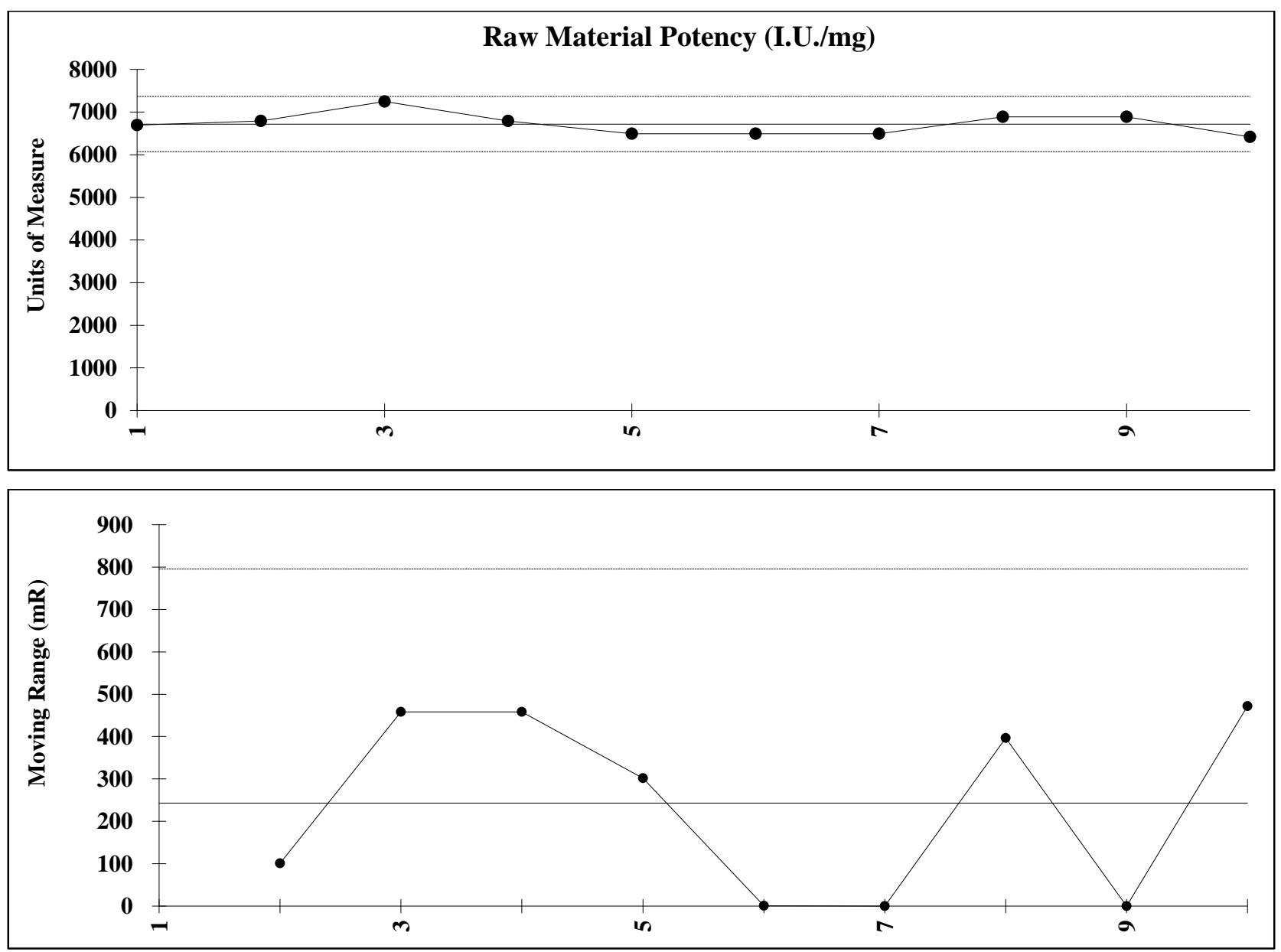

Figure 7. I-MR (XmR) control charts for potency determinations of Nystatin in the pharmaceutical preparations and as raw materials

On the other hand, the preparation value terms exceeded the warning value which is consistent with the noticeable deviations of the estimated potencies for unknown Nystatin products in some tests from the standard activity which in turn is reflected in the control chart. The possibility of the existence of a non-detectable slight curvature cannot also be ruled out. Importantly, the compendial requirements have two basic requirements for a successful analysis in this case [26]:

- Linear regression which should be fairly higher than the warning value.

- Non-parallelism which should be lower than the critical value.

Non-official requirements of the variance ratios that could be added to the causes of the experimental variability include all the remaining sources of variations which embrace preparation, row and column totals and they are informative for the analysts to track the significance of the sources of variations to improve them and enhance the assay conditions. The estimated potencies of FN and RM products showed an initial acceptable trend that lies within the control limits. However, a larger number of recorded test results (e.g. $\geq 50$ ) are needed to build a meaningfully solid trend to trace the analysis behaviour over the long term. The analysis is generally acceptable yielding valid results, but caution should be taken to improve test conditions, notably variations due to preparation, raw and column factors. Construction of control charts could be rendered easy by loading the needed equations in a spreadsheet and regularly maintaining a routine update of data to follow the change in the inspection characteristics chronologically [43]. This will further assist in the management of the quality of the microbiological analysis system in the laboratory.

\section{Conclusion}

Despite the great advancement in the automation of the laboratory workflow which renders the activities more consistent, predictable and reproducible, this privilege could not be attained smoothly globally. Expensive and sophisticated technology is always a barrier that hinders the progress in the developing countries to catch that in the developed nations. Microbiological analysis is cheap, simple and does not require extensive utilities and instrumentation (if compared with other techniques such HPLC or UPLC methods), yet it is largely dependent on the training and skills of the analysts. Thus, quantitative metrics for judgment of the test validity are crucial and mandatory to be established based on statistical interpretation before accepting the output results which affect product quality and decision-making. The statistical analysis for the sources of variations and errors in the potency determination coupled with trending charts would be of great importance for spotting any deviations or abnormal drifts in the quality of the analysis performed and its routs early enough before any excursions could devastate the outcome results and conclusions derived from the test. 
Preliminary assessment of the quality of the microbiological antibiotic assay is an indispensable step in the evaluation of the analysis before considering the calculated potency for the determination of the strength of RM or FN products. This initial investigation step is rendered easy and convenient through programming spreadsheets with formulae and equations for the calculation of the parameters that signify the quality of antibiotic assay, in addition to the potency estimation. Accordingly, the consistency and reproducibility of the performance of the microbiology laboratory and the analysed subjects could be assessed quantitatively over the long run using trending charts which also could be generated through Excel worksheets. Consequently, GLP could be ensured through a continuous improvement program by investigating the standard procedures, quality of reagents/media batches, instrument qualification/calibration and training of personnel.

\section{Declarations}

\subsection{Author Contributions}

Conceptualization; D.E. and M.E.; methodology; M.E.; software; E.R.; validation; M.E.; E.R. and D.E.; formal analysis; M.E.; investigation; E.R.; resources; D.E.; data curation; E.R.; writing — original draft preparation; M.E.; writing-review and editing; D.E.; visualization; E.R.; supervision; D.E.; project administration; E.R.; funding acquisition; D.E. All authors have read and agreed to the published version of the manuscript.

\subsection{Funding}

The authors received no financial support for the research, authorship, and/or publication of this article.

\subsection{Ethical Approval}

Not applicable, because this article does not contain any studies with human or animal subjects.

\subsection{Data Availability Statement}

The data presented in this study are available in article.

\subsection{Conflict of Interest}

The authors declare that they have no known competing financial interests or personal relationships that could have appeared to influence the work reported in this paper.

\section{References}

[1] Caffrey, P., De Poire, E., Sheehan, J., \& Sweeney, P. (2016). Polyene macrolide biosynthesis in streptomycetes and related bacteria: recent advances from genome sequencing and experimental studies. Applied Microbiology and Biotechnology, 100(9), 3893-3908. doi:10.1007/s00253-016-7474-z.

[2] Lyu, X., Zhao, C., Hua, H., \& Yan, Z. (2016). Efficacy of nystatin for the treatment of oral candidiasis: a systematic review and meta-analysis. Drug Design, Development and Therapy, 1161. doi:10.2147/dddt.s100795.

[3] C. Johnson; T. (2021). What is Candidiasis?. WebMD. Availble online: https://www.webmd.com/skin-problems-andtreatments/guide/what-is-candidiasis-yeast-infection (accessed on April 2021).

[4] Kaufman, D., Liken, H., \& Odackal, N. J. (2019). Diagnosis, Risk Factors, Outcomes, and Evaluation of Invasive Candida Infections. Infectious Disease and Pharmacology, 69-85. doi:10.1016/b978-0-323-54391-0.00007-2.

[5] Millsop, J. W., \& Fazel, N. (2016). Oral candidiasis. Clinics in Dermatology, 34(4), 487-494. doi:10.1016/j.clindermatol.2016.02.022.

[6] Ozturk, M. A., Gunes, T., Koklu, E., Cetin, N., \& Koc, N. (2006). Oral nystatin prophylaxis to prevent invasive candidiasis in Neonatal Intensive Care Unit. Mycoses, 49(6), 484-492. doi:10.1111/j.1439-0507.2006.01274.x.

[7] Young, G. A. R., Bosly, A., Gibbs, D. L., \& Durrant, S. (1999). A double-blind comparison of fluconazole and nystatin in the prevention of candidiasis in patients with leukaemia. European Journal of Cancer, 35(8), 1208-1213. doi:10.1016/s09598049(99)00102-1.

[8] National Center for Biotechnology Information (2021). PubChem Compound Summary for CID 138403272. Available online: https://pubchem.ncbi.nlm.nih.gov/compound/138403272 (accessed on October 2021).

[9] Lancelin, J. M., \& Beau, J. M. (1995). Stereostructure of glycosylated polyene macrolides: the example of pimaricin. Bulletin de la Société chimique de France, 2(132), 215-223.

[10] Martin, J. F., \& McDaniel, L. E. (1977). Production of Polyene Macrolide Antibiotics. Advances in Applied Microbiology Volume 21, 1-52. doi:10.1016/s0065-2164(08)70037-6. 
[11] Keller; M. D. (2017). The effect of amphotericin B on yeast growth; \& the isolation; \& identification of sterols for protozoan protein X-ray crystallography (Doctoral dissertation). Available online: https://tu-ir.tdl.org/handle/2346/72716 (accessed on October 2021).

[12] Dafale, N. A., Semwal, U. P., Agarwal, P. K., Sharma, P., \& Singh, G. N. (2015). Development and validation of microbial bioassay for quantification of Levofloxacin in pharmaceutical preparations. Journal of Pharmaceutical Analysis, 5(1), 18-26. doi:10.1016/j.jpha.2014.07.007.

[13] Pfaller, M. A., Krogstad, D. J., Granich, G. G., \& Murray, P. R. (1984). Laboratory evaluation of five assay methods for vancomycin: bioassay, high-pressure liquid chromatography, fluorescence polarization immunoassay, radioimmunoassay, and fluorescence immunoassay. Journal of Clinical Microbiology, 20(3), 311-316. doi:10.1128/jcm.20.3.311-316.1984.

[14] Greco; G. M. (1998). Microbiological assay systems for the analysis of antibiotics in pharmaceutical formulations (Ph.D. Dissertation). The State University Of New Jersey; USA;

[15] Pinto; T. J. A.; Lourenco; F. R.; \& Kaneko; T. M. (2007). Microbiological assay of Gentamycin employing an alternative experimental design. In AOAC Annual Meeting; \& Exposition. Vol. 121; p. 157.

[16] Saviano, A. M., Francisco, F. L., \& Lourenço, F. R. (2014). Rational development and validation of a new microbiological assay for linezolid and its measurement uncertainty. Talanta, 127, 225-229. doi:10.1016/j.talanta.2014.04.019.

[17] Dafale, N. A., Agarwal, P. K., Semwal, U. P., \& Singh, G. N. (2013). Development and validation of microbial bioassay for the quantification of potency of the antibiotic cefuroxime axetil. Anal. Methods, 5(3), 690-698. doi:10.1039/c2ay25848j.

[18] Cazedey, E. C. L., \& Salgado, H. R. N. (2011). Development and Validation of a Microbiological Agar Assay for Determination of Orbifloxacin in Pharmaceutical Preparations. Pharmaceutics, 3(3), $572-581$. doi:10.3390/pharmaceutics3030572.

[19] Yamamoto, C. H., \& Pinto, T. J. A. (1996). Rapid Determination of Neomycin by a Microbiological Agar Diffusion Assay Using Triphenyltetrazolium Chloride. Journal of AOAC International, 79(2), 434-440. doi:10.1093/jaoac/79.2.434.

[20] Lourenço, F. R., \& Pinto, T. de J. A. (2009). Comparison of three experimental designs employed in gentamicin microbiological assay through agar diffusion. Brazilian Journal of Pharmaceutical Sciences, 45(3), 559-566. doi:10.1590/s1984-82502009000300022.

[21] Hovstadius, B., Åstrand, B., \& Petersson, G. (2009). Dispensed drugs and multiple medications in the Swedish population: an individual-based register study. BMC Clinical Pharmacology, 9(1). doi:10.1186/1472-6904-9-11.

[22] Dafale, N. A., Semwal, U. P., Rajput, R. K., \& Singh, G. N. (2016). Selection of appropriate analytical tools to determine the potency and bioactivity of antibiotics and antibiotic resistance. Journal of Pharmaceutical Analysis, 6(4), $207-213$. doi:10.1016/j.jpha.2016.05.006.

[23] Nam, J.-H., Shin, J.-H., Kim, T.-H., Yu, S., \& Lee, D.-H. (2019). Comparison of biological and chemical assays for measuring the concentration of residual antibiotics after treatment with gamma irradiation. Environmental Engineering Research, 25(4), 614-621. doi:10.4491/eer.2019.270.

[24] Nahar, S., Khatun, M. S., \& Kabir, M. S. (2020). Application of microbiological assay to determine the potency of intravenous antibiotics. Stamford Journal of Microbiology, 10(1), 25-29. doi:10.3329/sjm.v10i1.50729.

[25] Hewitt; W. (2004). Microbiological assay for pharmaceutical analysis: a rational approach. CRC press. doi:10.1201/b12428.

[26] European Pharmacopoeia Commission. (2004). Statistical analysis of results of biological assays; \& tests. European Pharmacopoeia; 571-600.

[27] United States Pharmacopoeia (USP); \& National Formulary (NF). (2021). «81〉 Antibiotics-Microbial assays; USP43-NF38; US Pharmacopeial Convention Inc.; Rockville; p. 86-93.

[28] Loureno, F. R., Kaneko, T. M., \& Pinto, T. D. J. A. (2007). Validation of Erythromycin Microbiological Assay Using an Alternative Experimental Design. Journal of AOAC International, 90(4), 1107-1110. doi:10.1093/jaoac/90.4.1107.

[29] Eissa, M. E. A. (2018). Microbiological quality of purified water assessment using two different trending approaches: A case study. Sumerianz Journa 1 of Scientific Research, 1(3), 75-79.

[30] Eissa, M. E., \& Abid, A. M. (2018). Application of statistical process control for spotting compliance to good pharmaceutical practice. Brazilian Journal of Pharmaceutical Sciences, 54(2). doi:10.1590/s2175-97902018000217499.

[31] Rashed; E. R.; \& Eissa; M. E. (2020). Long-term monitoring of Cancer Mortality Rates in USA: A descriptive analysis using statistical process control tools. Iberoamerican Journal of Medicine; 2(2); 55-60. doi:10.5281/zenodo.3740610.

[32] Eissa; M. E. (2018). Application of attribute control chart in the monitoring of the physical properties of solid dosage forms. Journal of Progressive Research in Modern Physics; \& Chemistry (JPRMPC); 3(1); 104-113. 
[33] Mohamed; R. A.; \& Mariam; H. M. (2017). Using Completely Randomized Design of Parallel Linear Model for Estimating the Biological Potency of Human Insulin Drugs: An Empirical Study. Biostatistics \& Biometrics Open Access Journal; 3(4); $115-121$.

[34] Mairesse, A., Wauthier, L., Courcelles, L., Luyten, U., Burlacu, M., Maisin, D., ... Gruson, D. (2021). Biological variation and analytical goals of four thyroid function biomarkers in healthy European volunteers. Clinical Endocrinology, 94(5), 845-850. doi:10.1111/cen.14356.

[35] Cochran, W. G. (1951). Testing a Linear Relation among Variances. Biometrics, 7(1), 17. doi:10.2307/3001601.

[36] European Pharmacopoeia (2002) 4th Ed.; Council of Europe; Strasbourg; France.

[37] Swift, M. L. (1997). GraphPad Prism, Data Analysis, and Scientific Graphing. Journal of Chemical Information and Computer Sciences, 37(2), 411-412. doi:10.1021/ci960402j.

[38] Motulsky; H. J. (2003). Prism 4 statistics guide — statistical analyses for laboratory \& clinical researchers. GraphPad Software Inc.; San Diego; CA; 122-126.

[39] Eissa; M. E. (2018). Variable \& attribute control charts in trend analysis of active pharmaceutical components: Process efficiency monitoring \& comparative study. Experimental Medicine (EM); 1(1); 32-44.

[40] Eissa; M. (2018). Evaluation of microbiological purified water trend using two types of control chart. European Pharmaceutical Review; 23(5); 36-38.

[41] Sullivan, J. H., \& Woodall, W. H. (1996). A Control Chart for Preliminary Analysis of Individual Observations. Journal of Quality Technology, 28(3), 265-278. doi:10.1080/00224065.1996.11979677.

[42] Fatimah, Sayuti, M., \& Pertiwi, E. P. (2018). Quality control of palm kernel oil using Individual Moving Range (I-MR) chart. MATEC Web of Conferences, 204, 01006. doi:10.1051/matecconf/201820401006.

[43] Henderson; G. R. (2011). Six Sigma quality improvement with MINITAB. John Wiley \& Sons. 